\title{
Socio-Ecological Network Structures from Process Graphs
}

\author{
Angelyn Lao ${ }^{1 *}$, Heriberto Cabezas ${ }^{2}$, Ákos Orosz ${ }^{2}$, Ferenc Friedler ${ }^{2}$, Raymond Tan $^{3}$ \\ 1 Mathematics and Statistics Department, De La Salle University, 2401 Taft Avenue, \\ 0922 Manila, Philippines
}

2 Institute for Process Systems Engineering and Sustainability, Pázmány Péter Catholic University, Szentkirályi utca 28, 1088 Budapest, Hungary

3 Chemical Engineering Department, De La Salle University, 2401 Taft Avenue, 0922

Manila, Philippines

*Corresponding author

E-mail: angelyn.lao@dlsu.edu.ph

\section{Abstract}

We propose a process graph (P-graph) approach to develop ecosystem networks from knowledge of the properties of the component species. Originally developed as a process engineering tool for designing industrial plants, the P-graph framework has key advantages over conventional ecological network analysis (ENA) techniques. A P-graph is a bipartite graph consisting of two types of nodes, which we propose to represent components of an ecosystem. Compartments within ecosystems (e.g., organism species) are represented by one class of nodes, while the roles or functions that they play relative to other compartments are represented by a second class of nodes. This bipartite graph representation enables a powerful, unambiguous representation of relationships among ecosystem compartments, which can come in tangible (e.g., mass flow in predation) or intangible form (e.g., symbiosis). For example, within a P-graph, the distinct roles of bees as pollinators for some plants and as prey for some animals can be explicitly represented, which would not otherwise be possible using conventional ENA. After a discussion of the mapping of ecosystems into P-graph, we also discuss how this 
framework can be used to guide understanding of complex networks that exist in nature. Two component algorithms of P-graph, namely maximal structure generation (MSG) and solution structure generation (SSG), are shown to be particularly useful for ENA. This method can be used to determine the (a) effects of loss of specific ecosystem compartments due to extinction, (b) potential efficacy of ecosystem reconstruction efforts, and (c) maximum sustainable exploitation of human ecosystem services by humans. We illustrate the use of P-graph for the analysis of ecosystem compartment loss using a small-scale stylized case study, and further propose a new criticality index that can be easily derived from SSG results.

\section{Author summary}

In this study, we propose the novel application of the process graph (P-graph) methodology to the analysis of ecological networks. P-graph was originally developed for engineering design problems; in our work, we show how its five axioms and two algorithms - maximal structure generation (MSG) and solution structure generation (SSG) can be adapted to the problem of understanding complex interactions in ecosystems. The methodology allows multiple types of interactions among ecosystem components to be handled simultaneously based on representation as a bipartite graph. Complete network structures can be deduced from knowledge of local interactions of components using MSG. Finally, all structurally feasible networks of viable ecosystems can be identified with SSG. We illustrate the features of the P-graph methodology with a stylized illustrative example.

\section{Introduction}

Mathematical models have proven to be valuable and useful tools for the analysis of ecological networks and their emergent properties. Early examples include input-output models similar to those used to describe economic structures [1]. Metrics to describe the structure of ecological networks naturally flowed from the use of such quantitative tools (e.g., [2]). These tools provide a lens for the analysis of complex interactions that arise from interactions among ecosystem components. In many cases, specialists only fully 
understand local interactions of ecosystem components, and thus need modelling techniques such as ecological network analysis (ENA) to deduce high-level interactions that occur through direct and indirect linkages.

Searching the Scopus database using "ecological network analysis" as a search term yields 429 published documents, over half of which were published from 2016 to the present. Despite the broad array of techniques already used in ENA, according to Poisot et al. [3], "ecology will probably continue to benefit from those tools, metrics and models developed in other fields." Thus, in this paper, we discuss a potential new tool for ENA.

The use of network-based techniques for the analysis of social-ecological interdependencies remains a challenge [4]. Such models are extended from ENA methods through linkage with a network model of a human community at an appropriate scale. Network techniques are useful for understanding emergent behavior that arises from complex interactions among system components [5]. Such system-level behavior is often not immediately evident from the local properties of individual components, and failure to account for them can often lead to unexpected results [3].

On the other hand, judicious use of ENA and extensions that link them to man-made systems can provide useful insights for sustainable use or resources and ecosystem services [6-9]. These services refer to the conditions and processes by which ecosystems sustain and fulfill human life [10], which can be achieved for example through the provision of shelter, nectar, alternative prey/host and/or pollen for natural enemies which can be deployed by humans $[11,12]$. The insights drawn can be used to guide decisions on ecosystem conservation or restoration measures. By incorporating ecological-economic interactions, the models can also be used to estimate the limits of exploitation of ecosystem products and services.

Two challenges are apparent in the current literature on social-ecological models. First, existing techniques must assume that a single type of interaction predominates in the system. For example, trophic linkages in food webs are the most commonly represented type of relationship in ecological network models. In order to better understand the behavior of real ecosystems, the capability to represent the existence of multiple simultaneous interdependencies is needed [13]. The current approach relies on multiplex network modelling approaches - i.e., the use of multiple linked network models, each representing one type of interdependence $[4,14]$. 
The second challenge is network assembly. Typically, model developers use knowledge of local interactions of system components, coupled with heuristic network assembly rules, to deduce the structure of the network [15]. While this technique appears to work reasonably well, a mathematically rigorous approach to network assembly can improve ecological modelling by eliminating the potential for human error and biases that always exists when a heuristic is used.

The structure of even relatively small ecosystems can be rather complex. Any interactions that could not be observed will, therefore, often not be included. One should also note that frequently more is known about the individual species making up the ecosystem rather than the structure of the ecosystem itself. For example, zoologists are usually able to identify staple food sources of animal species with good accuracy. The reason is that it is far easier to study one species at a time than many species simultaneously. Most ENA work then gives fragmented or one-dimensional representations of real ecosystems. Whereas in reality, ecosystem components play multiple roles relative to each other; thus, there is a need for a framework that allows concurrent modelling of these multiple roles and their resulting complex interactions [16].

While there has been a concerted effort by a small number of researchers to be able to track and measure the complexity of ecological systems, the common approaches in systems ecology still limit their focus to biomass (or energy, nutrients, etc.) fluxes between species (nodes), recycling of material, decomposition, or production. The species are treated as compartments that are interconnected by transaction of the energy-matter substance flowing between them. More subtle interactions such as, for example, the provision of shelter are difficult to include within this framework.

We propose the use of a class of models known as process graphs to deal with these difficulties. The process graph (or P-graph) framework was originally developed as a graph theoretic technique for handling combinatorial challenges in industrial plant design $[17,18]$. In such engineering problems, the typical task is to design an optimal plant subject to economic constraints to produce a set of specified products from available raw materials, using a predefined set of candidate conversion processes. These process units must be assembled into a feasible network of conversion processes that can be then translated into a viable industrial plant. This problem is known as process 
network synthesis (PNS).

In PNS, a P-graph is a bipartite graph with two sets of nodes used to represent processes and streams. Arcs are used to denote the relationship of the inputs and outputs of material or energy streams into each of the candidate processes. The key feature of the P-graph framework is the availability of algorithms [18] developed rigorously based on a set of axioms that apply to all PNS problems [17]. For any given plant design task, these algorithms can be used to automatically generate a maximal structure that represents the union of all possible process networks, and also to identify every structurally feasible process network. They eliminate the risk of human error or bias in specifying an incomplete process network during engineering design.

The P-graph framework has been applied to a wide range of PNS problems, as well as to other engineering problems of analogous form [19]. But the basic mathematical machinery is independent of the nature of the nodes and links between the nodes, and it is, therefore, applicable in principle to any network. However, there is no record of its use for ecological systems in the scientific literature.

P-graph can be used to represent linked social-ecological systems. Various agents and compartments can be represented as process nodes (O-type nodes), as in conventional network approaches. These nodes can be linked to each other via different types of relationships, such as predation among species or consumption of resources and ecosystem services by humans. The different roles or functions played by the process nodes in the network can then be represented as intermediate nodes (M-type nodes). In P-graph the arcs never link process nodes directly to each other; instead, the arcs are linked to intermediate nodes to unambiguously specify the exact nature of the relationship between any two components of the social-ecological network. This feature allows different types of interactions to be represented in a single graph, which is a departure from the conventional multiplex graph approach $[4,14]$.

In addition to enabling a more elegant system representation, the P-graph framework can also be used to generate a complete social-ecological network based only on knowledge of local interactions of each network component. The maximal structure generation (MSG) and solution structure generation (SSG) algorithms can replace the conventional heuristic approach which still runs the risk of yielding an incorrect or incomplete or biased network [15]. The capability to identify all structurally feasible 
networks for a given set of compartments and agents has many potential applications in $\quad 104$ sustainable ecosystem management. For example, it becomes possible to identify which ${ }_{105}$ structures can be functional, and which ones will collapse. Keystone species can be $\quad{ }_{106}$ identified based on the frequency of occurrence in different solution structures. In direct $\quad{ }_{107}$ analogy with the industrial plant design problem for which P-graph was originally developed, it is possible in principle to determine the minimum ecological cost of providing a given set of resource outputs and ecosystem services for a community of humans. This type of problem can be applied concretely to cases such as the management of fisheries, forests, and the like. The list of other possible problems extends well beyond these examples.

We show the rich potential of the P-graph to deal with the analysis of a simple social-ecological network model. This technique has already proven to be a powerful tool for various engineering design problems, and it has features which can be useful to deal with challenges that are difficult to handle using conventional network techniques.

\section{Materials and methods}

\section{Process graph}

The P-graph framework is a graph theoretic approach to PNS problems encountered during the design of industrial plants [17] as previously mentioned. In such problems, the challenge is to determine a suitable (and preferably optimal) design for the manufacture of a set of products, given available raw materials, capital, and component process units. The P-graph framework (e.g. Fig 1) uses a bipartite graph consisting of M-type nodes (circles) to represent materials, and O-type nodes (horizontal bars) to represent operating units; arcs are then used to signify how materials relate to processes as either inputs or outputs. For example, using Fig 1, O-type node 'O1' needs M-type node 'A' in order to provide M-type node ' $\mathrm{E}$ '. If the M-type node is produced by more than one O-type nodes, it can be produced by one, by another one, or any combination of them. For example, M-type node 'E' can be produced by O-type node 'O1', 'O2', or both of them. In a P-graph, inputs to an O-type node are necessary. For example, M-type nodes ' $E$ ' and ' $F$ ' are both necessary inputs to O-type node 'O4'. This makes 
O-type nodes $\mathrm{O} 2$ and $\mathrm{O} 3$ to also be essential. As discussed below, these concepts can be ${ }^{133}$ adapted to represent ecological networks.

Fig 1. P-graph representation of the process structure (lifted from [20]).

The P-graph framework consists of three component algorithms developed rigorously based on five axioms that serve as core assumptions of all PNS problems [17]. The axioms can be modified as shown in Table 1 to fit the context of ecological modelling. An ecosystem is assumed to consist of components known as ecosystem functional units, ${ }_{138}$ which are analogous to operating units or processes in engineering design problems. $\quad{ }_{139}$ These ecosystem functional units are represented as O-type nodes. On the other hand, various ecosystem services that are analogous to material streams in engineering design problems are all represented as M-type nodes. Some ecosystem services can be classified as exogenous (i.e., originating entirely from outside the ecosystem boundary) and are analogous to raw materials for industrial plants; other ecosystem services are classified as terminal (i.e., exiting the ecosystem boundary to be utilized by humans) and correspond to industrial final products. Ecosystem services that are neither exogenous nor terminal act as intermediates and are produced and consumed entirely within the ecosystem network without crossing its boundaries.

The MSG algorithm rigorously generates a network which is the union of all structurally or combinatorially feasible networks that can be generated from the component units [21]. The SSG is capable of generating all combinatorially feasible networks, based on total enumeration of possible structures arising from localized relationships between materials and processes or services and functional units in the case of ecosystems [18]. Combinatorial feasibility is based solely on network connectivity and does not account for stream flow rates or quantities. The accelerated branch-and-bound algorithm (ABB) can then be used to effectively determine optimal and near-optimal solutions to PNS problems by evaluation of the performance of candidate networks, while excluding infeasible and redundant solutions from the search strategy. This effectiveness is derived from the use of implicit information embedded in all PNS problems [22]. A recent review paper gives a comprehensive survey of P-graph literature focusing on engineering applications [19]. The potential applicability of this framework to deal with PNS-like problems in generalized networks - such as economic

135 136 137 140 141 142 143 144 145 146 147 148 149 150 151 152 153 154 155 156 157 158 159 160 161 162 
systems or organizational structures - is discussed by [23].

Table 1. P-graph Axioms.

\begin{tabular}{|l|l|}
\hline Process Engineering & Socio-Ecological Systems \\
\hline $\begin{array}{l}\text { (S1) Every final product is represented } \\
\text { in the structure. }\end{array}$ & $\begin{array}{l}\text { (SE1) There should be at least one well- } \\
\text { defined terminal ecosystem service in } \\
\text { the ecosystem structure. }\end{array}$ \\
\hline $\begin{array}{l}\text { (S2) A material represented in the struc- } \\
\text { ture is a raw material if and only if it } \\
\text { is not an output of any operating unit } \\
\text { represented in the structure. }\end{array}$ & $\begin{array}{l}\text { in the structure is exogenous if and only } \\
\text { if is not an output of a functional unit } \\
\text { defined in the ecosystem structure. }\end{array}$ \\
\hline $\begin{array}{l}\text { (S3) Every operating unit represented in } \\
\text { the structure is defined in the synthesis } \\
\text { problem. }\end{array}$ & $\begin{array}{l}\text { (SE3) Every ecosystem functional unit } \\
\text { in the ecosystem structure is well de- } \\
\text { fined. }\end{array}$ \\
\hline $\begin{array}{l}\text { (S4) Any operating unit represented in } \\
\text { the structure has at least one path lead- } \\
\text { ing to a product. }\end{array}$ & $\begin{array}{l}\text { (SE4) Any ecosystem functional unit has } \\
\text { at least one path leading to a terminal } \\
\text { ecosystem service. }\end{array}$ \\
\hline $\begin{array}{l}\text { (S5) If a material belongs to the struc- } \\
\text { ture, it must be an input to or output } \\
\text { from at least one operating unit repre- } \\
\text { sented in the structure. }\end{array}$ & $\begin{array}{l}\text { SE5) If an ecosystem service belongs } \\
\text { an input to or output from at least one } \\
\text { ecosystem functional unit represented } \\
\text { in the structure. }\end{array}$ \\
\hline $\begin{array}{l}\text { Assumption: capital is available to } \\
\text { pay for operating units and operating } \\
\text { costs. }\end{array}$ & $\begin{array}{l}\text { Assumption: energy is available to } \\
\text { keep the ecosystem structure function- } \\
\text { ing. }\end{array}$ \\
\hline $\begin{array}{l}\text { Goals: (1) meet production goal at (2) } \\
\text { minimum cost for the structure and op- } \\
\text { eration. }\end{array}$ & $\begin{array}{l}\text { Goals: meet ecosystem services goal; } \\
\text { and (2) minimize a cost metric (e.g. } \\
\text { money, ecological footprint, energy, etc.) } \\
\text { for the structure, management, func- } \\
\text { tional, and operations of the ecosystem } \\
\text { services. }\end{array}$ \\
&
\end{tabular}

The proposal of new ecological networks (established from an ecosystem) and the discussion of their feasibility can be structured with the application of P-graph, to perform a combinatorially complete search of ecological interactions. By imposing constraints, the search algorithm allows us to compose feasible pathways. These constraints include mass balances and the existence of known ecological interactions such as pollination or provision of shelter. The search domain to find feasible ecological interactions in the ecosystem can be reduced by eliminating or replacing species/entities. No feasible ecological interactions are lost in this process.

\section{P-graph software and toolbox}

The software P-graph Studio is hosted and supported by the Department of Computer Science and Systems Technology at the University of Pannonia [20]. This software can 173 174 
be accessed and used free of charge for research purposes via www.p-graph.org. It is $\quad 175$ currently on version 5.2.2.2 and can only run under Microsoft Windows. The following 176 prerequisites are required: Microsoft .NET Framework 4.5.1 (x86 and x64) and Windows ${ }_{177}$ Installer 4.5. Since P-graph Studio is designed for use on engineering design problems, 178 this article comes with a prototype code implemented in Microsoft Excel and Visual 179 Basic for Windows (VBA) which is more readily used for the analysis of social-ecological ${ }_{180}$ networks. Using the attached code (S1 Appendix \& S2 Appendix), interested readers $\quad{ }_{181}$ can replicate the case study that follows, modify it, or even analyze new examples. $\quad{ }_{182}$

\section{Results}

\section{A simple socio-ecological system}

This section illustrates the use of P-graph on socio-ecological systems via a case study; ${ }_{185}$ the analysis shown here focuses purely on structural aspects and does not delve into stream flow rates. Energy usually enters ecosystems as sunlight and is captured in 186 chemical form by photosynthesizes like plants and algae (see Fig 2). The energy is then passed through the ecosystem, changing form as organisms metabolize, produce waste, consume one another, and eventually, die and decompose. Each time energy changes forms, some of it is converted to heat as required by the second law of thermodynamics. Heat still counts as energy - and thus no energy has been destroyed - but it generally cannot be used as an energy source by living organisms. Ultimately, energy that enters the ecosystem as sunlight is dissipated as heat and radiated back into space on global scale. This one-way flow of energy through ecosystems means that every ecosystem needs a constant supply of energy, usually from the sun, in order to maintain its order and function. Energy can be passed between organisms, but it cannot be completely recycled because some of it is lost as heat in each transfer.

The socio-ecological system (in Fig 2) is further elaborated in Fig 3A. In this system, we have defined specific characteristics for the plants and herbivores. For the plants, Plant 1 provides food for Herbivore 1 and Herbivore 2, and Habitat for herbivores and carnivores; Plant 2 also provides food for Herbivore 1 and Herbivore 2, and contributes to the Detritus; and Plant 3 contributes to the Detritus, and provides food for 
Herbivore 2 and humans. As for herbivores, both Herbivore 1 and Herbivore 2 contribute to the Detritus and provide food for Carnivore. Only Herbivore 1 contributes to the pollination, and only Herbivore 2 provides food for humans.

Fig 2. The flow of energy through a simple ecosystem. The energy flow from the sun is taken up by plants, some of which are eaten by herbivores, which, in turn, are eaten by carnivores. Part of the energy flows to the outside of the system.

\section{Socio-ecological P-graph (network) structure}

The stylized ecosystem shown in Fig 3A can be translated into P-graph form as illustrated in Fig 3B. The representation of final output of the ecosystem (i.e. food extracted by humans) illustrates the use of Axiom (SE1) in Table 1. Likewise, representation of solar energy as an external input is based on Axiom (SE2). All compartments (Plant1, Plant2, Plant3, Herbivore1, Herbivore2, Carnivore, Soil_microbes) are specified in the model as O-type nodes at the relevant level of resolution, based on Axiom (SE3). The inputs and outputs into each of these compartments signify their requirements and their contributions to the ecosystem, respectively, and they are represented as M-type nodes (Habitat, Pollination, Soil_nutrients, Detritus, Food_for_Carnivore, Food_for_Herbivore1, Food_for_Herbivore2). For example, herbivores draw on plant matter for food and also need shelter provided by vegetation in their habitat; in turn, they provide food to carnivores and humans, and also propagate seeds of plants. These inputs and outputs into the compartments are defined based only on local information; generation of the ecosystem network can be done algorithmically in P-graph via MSG. It can easily be seen in Fig 3 that the given structure also satisfies Axioms (SE4) and (SE5) in Table 1. That means that each functional unit has a path or contribution to the terminal ecosystem services (SE4), and all ecosystem services are inputs or outputs from a functional unit.

Fig 3. P-graph of the socio-ecological system. (A) Box diagram of the extended socio-ecological system illustrated in Fig 2. (B) P-graph of the same extended socio-ecological system shown in (A). This represents Structure S1, the maximal and most redundant structure in Table 2. 


\section{Discussion}

Given the P-graph structure in Fig 3B, twenty structures (S1-S20) can be generated. They are summarized in Table 2 and are illustrated in supplementary information S3

Figure. Interestingly, it can be observed that for this particular ecosystem, Carnivore

is not essential in the sense that the rest of the ecosystem is capable of producing the terminal services without it. This can be observed if the structures in Table 2 are compared pairwise - the odd-numbered structures contain Carnivore whereas the even-numbered structures do not. In other words, with or without Carnivore, the ecosystem seems to still function. Unlike the plants (Plant1, Plant2, Plant3) and herbivores (Herbivore1, Herbivore2), there is only one type of carnivore defined in the system. Without Carnivore, the node representing Food_for_carnivores will be reclassified as a terminal component, which may lead to overpopulation of some herbivores. The fact that Carnivore can be not essential is a consequence of excluding the carnivores as a source of Food_for_humans, hence removing their direct contribution to the terminal ecosystem service of Food_for_humans. Note that there is no arc connecting Carnivore to the Food_for_humans. Based on the defined social-ecological system (Fig 2), if humans only eat plants and herbivores, the existence of the carnivores is not essential.

The maximal and minimal structures are S1 (Fig 3B is also Fig 1A of S3 Figure) and S20 (Fig 4B), respectively. The maximal structure S1 contains all the components and entities defined in the social-ecological system (Fig 3B). In the minimal structure S20 (shown in Fig 4B), Plant1, Plant3, Herbivore2, and Carnivore are excluded from the system. This is a "bare bones" system with the absolute minimum functional units necessary to produce the terminal service of Food_for_humans. Fig 4A shows structure S10 from Table 2 which is an intermediate between the maximal structure (S1) and the minimal structure (S20). It can be seeing that structure S10 has some redundancy to provide food to humans from plant P1 or herbivore H2. This will, therefore, give it some ability to manage perturbations such as for example, a decrease in the population of plant P2 which in the minimal structure(S20) is the only source of food for humans.

Finally, note that for the plants, at least one of Plant1, Plant2, and Plant3 should be included in the system. If two plant types are to be excluded in the system at the

230 231 232 233 234 235 236 237 238 239 240 241 242 243 244 245 246 247 248 249 250 251 252 253 254 255 256 257 
Table 2. The ecosystem functional units (EFU) contained in each of the 20 structures.

\begin{tabular}{|c|c|c|c|c|c|c|c|}
\hline $\begin{array}{c}\text { Structure } \\
\text { Label }\end{array}$ & Plant1 & Plant2 & Plant3 & Herbivore1 & Herbivore2 & Carnivore & $\begin{array}{c}\text { Soil } \\
\text { microbes }\end{array}$ \\
\hline S1 & $\checkmark$ & $\checkmark$ & $\checkmark$ & $\checkmark$ & $\checkmark$ & $\checkmark$ & $\checkmark$ \\
\hline S2 & $\checkmark$ & $\checkmark$ & $\checkmark$ & $\checkmark$ & $\checkmark$ & & $\checkmark$ \\
\hline S3 & $\checkmark$ & $\checkmark$ & $\checkmark$ & $\checkmark$ & & $\checkmark$ & $\checkmark$ \\
\hline S4 & $\checkmark$ & $\checkmark$ & $\checkmark$ & $\checkmark$ & & & $\checkmark$ \\
\hline S5 & $\checkmark$ & & $\checkmark$ & $\checkmark$ & & $\checkmark$ & $\checkmark$ \\
\hline S6 & $\checkmark$ & & $\checkmark$ & $\checkmark$ & & & $\checkmark$ \\
\hline S7 & $\checkmark$ & & $\checkmark$ & $\checkmark$ & $\checkmark$ & $\checkmark$ & $\checkmark$ \\
\hline S8 & $\checkmark$ & & $\checkmark$ & $\checkmark$ & $\checkmark$ & & $\checkmark$ \\
\hline S9 & $\checkmark$ & $\checkmark$ & & $\checkmark$ & $\checkmark$ & $\checkmark$ & $\checkmark$ \\
\hline S10 & $\checkmark$ & $\checkmark$ & & $\checkmark$ & $\checkmark$ & & $\checkmark$ \\
\hline S11 & $\checkmark$ & $\checkmark$ & & $\checkmark$ & & $\checkmark$ & $\checkmark$ \\
\hline S12 & $\checkmark$ & $\checkmark$ & & $\checkmark$ & & & $\checkmark$ \\
\hline S13 & & $\checkmark$ & $\checkmark$ & $\checkmark$ & $\checkmark$ & $\checkmark$ & $\checkmark$ \\
\hline S14 & & $\checkmark$ & $\checkmark$ & $\checkmark$ & $\checkmark$ & & $\checkmark$ \\
\hline S15 & & $\checkmark$ & $\checkmark$ & $\checkmark$ & & $\checkmark$ & $\checkmark$ \\
\hline S16 & & $\checkmark$ & $\checkmark$ & $\checkmark$ & & & $\checkmark$ \\
\hline S17 & & $\checkmark$ & & $\checkmark$ & $\checkmark$ & $\checkmark$ & $\checkmark$ \\
\hline S18 & & $\checkmark$ & & $\checkmark$ & $\checkmark$ & & $\checkmark$ \\
\hline S19 & & $\checkmark$ & & $\checkmark$ & & $\checkmark$ & $\checkmark$ \\
\hline S20 & & $\checkmark$ & & $\checkmark$ & & & $\checkmark$ \\
\hline
\end{tabular}

Fig 4. Minimal structure generated from the P-graph illustrated in Fig 3.

The 20 structures generated from P-graph can be found in S3 Figure. (A) Structure S10 is an intermediate structure between the maximal structure (S1) and the minimal structure (S20). (B) The minimal structure is Structure S20 in Table 2, it does not need Plant1, Plant2, Herbivore2, and Carnivore to provide the necessary service.

same time, Plant2 cannot be one of them. It can only be Plant1 and Plant3, as shown in Table 2. Removing Plant2 and Plant3 will directly affects the food provided for humans. Whereas, removing Plant1 and Plant2 directly affect the food provided for Herbivore1 and the habitat provided for the herbivore and carnivore. All these will disrupt the sustainability of the system and management of the ecosystem services.

In general, a functional ecosystem structure needs to always contain the following: at least one plant type (Plant1, Plant2, Plant3), at least one herbivore type

(Herbivore1, Herbivore2), and the soil_microbes. However, both Herbivore1 and soil_microbes are irreplaceable (they are present in all 20 structures) and appear to be crucial in the social-ecological system. It can be explained by the important components they provided, e.g. Soil_nutrients and Pollination, that are essential and needed by the plants (Plant1, Plant2, Plant3) to grow and live. 
There is one key distinction between this new application of P-graph and its original 270 purpose. In engineering PNS problems, the task is to generate possible networks from ${ }_{271}$ process building blocks as part of the design procedure. In this work, the ecosystem $\quad 272$ network already exists but the full structure may not be completely known to the human observer. P-graph thus serves two purposes. First, the overall network structure is deduced based on an understanding of the local properties of its components using MSG. Second, possible networks that can emerge from the present one are also deduced using SSG. These alternative networks represent the structurally viable ecosystems that can evolve from the current one due to human-induced or natural perturbations, e.g. the removal of a functional unit or biological species.

The capability to enumerate all combinatorially feasible networks or viable ecosystems via SSG also allows quantitative analysis of ecosystem components. Table $2{ }^{281}$ lists the 20 viable ecosystems for the illustrative case study. In practical ecosystem 282 management, it is necessary to identify critical components (e.g., keystone species) to enable prioritization of limited resources. The P-graph methodology can be used as a basis to derive new metrics. Here, we propose a criticality index which measures the importance of a particular ecosystem component to the whole system, as quantified by the frequency of its occurrence in the alternative networks enumerated with SSG.

The criticality index assumes a value in the interval $[0,1]$. An index 0 signifies a component which is unnecessary or expendable - at least from the perspective of providing ecosystem services to an external human population. In other words, a component with minimum criticality may or may not be present, without affecting the viability of the entire network. At the opposite extreme are functional components with criticality indices of 1 . They occur in all viable ecosystems and are thus indispensable components that provide essential ecosystems for which there are no alternative sources. The criticality index $\left(I C_{i}\right)$ for functional component or species $i$ is given by,

$$
I C_{i}=N_{i} / N
$$

where $N_{i}$ is the number of alternate structures containing functional component $i$, and $N$ is the total number of alternate viable structures under consideration. $N=20$ for the structures in Table 2. 

both have indices of 1 , being unique providers of pollination services and soil nutrients, 292 respectively. The other components in this ecosystem are of intermediate criticality. It ${ }_{293}$ can also be seen that the criticality of the individual components is dependent on the $\quad{ }^{294}$ structural redundancy or degeneracy of the ecosystem network; a small number of 295 critical components implies that the ecosystem is robust to disruptions. This means that system has the capacity to adjust to an alternate structure to compensate for the loss of a functional unit. Conversely, a large number of critical components implies that almost any loss of a component can be fatal to the overall system, i.e the system does not have any or has very few alternate structures to shift to compensate for the loss of functional units. This concept can be explored further in future developments. At this stage, the proposed criticality index can be used for the practical problem of prioritizing components for ecosystem management purposes. The question of the availability of feasible or viable structures that the system can shift to, gives rise to the concept of ecosystem brittleness or the converse concept of resilience. But this is an extensive topic which will be the subject of future research.

The P-graph framework offers the prospect of extending the toolbox of ecological modelling and brings two unique capabilities to bear. First, as a bipartite graph, P-graph allows unambiguous representation of multiple types of relationships that exist among compartments in a real ecosystem. This allows richer representation of ecosystems by enabling multiple roles and functions to be represented within a graph. Secondly, P-graph allows error-free generation of a complete network model based on local information about system components (via MSG), and furthermore allows all structurally feasible subsets of the complete network to be enumerated (via SSG). Such a capability overcomes the need to rely on heuristic assembly rules that are currently prevalent in ENA. The simple case study shown here focuses purely on structural features based on the existence of multi-functional links among compartments. The approach can be extended further to integrate ecological stoichiometry concepts [24], provided that the appropriate ratios are known. 


\section{Conclusion}

We propose the extension of the P-graph framework to ecosystem network structure and modeling. This novel application brings to bear specific features of P-graph to overcome the limitations of currently available tools (e.g., ENA). In particular, this framework allows (a) simultaneous representation of multiple types of relationships among ecosystem compartments; (b) rigorous generation of complete network models via MSG; and (c) elucidation of different feasible or functional ecosystems structures via SSG. The P-graph thus enables new insights to be drawn for purposes of effective management of ecosystem services, as illustrated here via a stylized ecosystem case study.

Further opportunities can be explored by using the optimization capability of P-graph for specific types of ecological modelling problems. The bulk of ENA literature focuses on the use of descriptive measures to understand the relative importance of compartments. However, in some problems, a specific objective function can be identified; for example, in the context of managing an ecosystem which is being altered or is being altered. This is important because ecosystems across the globe are being altered by human activity. The work may also be of scientific or policy interest to determine the maximum sustainable rate of withdrawal of renewable resources or ecosystem services from the environment. This case is a common issue in fisheries management. Conversely, there are cases where it will be necessary to determine the minimum land area needed to sustain a functional ecosystem, in response to loss of area due to habitat destruction by humans or by sea level rise. Other optimization problems in ecological modelling can be identified and solved in the future with the aid of P-graph via the MSG algorithm.

There are interesting prospects for future research in this new sub-area of systems ecology. The most promising directions are the direct application of the methodology to real ecosystems, and the hybridization of P-graph with established techniques such as ENA to enable better understanding of the behavior of ecological networks.

\section{Supporting information}


generate MSG \& SSG.

S2 Appendix. P-studio file. P-graph generated from P-studio.

S3 Figure. Structures generated from the P-graph. Twenty P-graph

\section{Acknowledgments}

This work was supported by the Pázmány Péter Catholic University Central Funds

Program, which allowed for the conduct of discussions among the authors of this manuscript.

\section{References}

1. Hannon B. The structure of ecosystems. J Theor Bio. 1973;41:535-46.

2. Finn JT. Measures of ecosystem structure and function derived from analysis of flows. J Theor Bio. 1976 Feb 1;56(2):363-80.

3. Poisot T, Stouffer DB, Kéfi S. Describe, understand and predict: why do we need networks in ecology?. Funct Ecol. 2016 Dec;30(12):1878-82.

4. Bodin Ö, Alexander SM, Baggio J, Barnes ML, Berardo R, Cumming GS, Dee LE, Fischer AP, Fischer M, Garcia MM, Guerrero AM. Improving network approaches to the study of complex social-ecological interdependencies. Nat Sustain. 2019 Jul;2(7):551-9.

5. Jordán F, Pereira J, Ortiz M. Mesoscale network properties in ecological system models. Curr Opin Syst Biol. 2019 Feb 1;13:122-8.

6. Cabezas H, Whitmore HW, Pawlowski CW, Mayer AL. On the sustainability of an integrated model system with industrial, ecological, and macroeconomic components. Resour Conserv Recycl. 2007 Apr 1;50(2):122-9.

7. Doshi R, Diwekar U, Benavides PT, Yenkie KM and Cabezas H. Maximizing sustainability of ecosystem model through socio-economic policies derived from 
multivariable optimal control theory. Clean Tech Env Policy. 2015 Aug 1;17(6):1573-83.

8. Fath BD. Systems ecology, energy networks, and a path to sustainability. Int J of Des Nat Ecodynamics. 2017 Jan 1;12(1):1-5.

9. Díaz S, Settele J, Brondízio ES, Ngo HT, Agard J, Arneth A, Balvanera P, Brauman KA, Butchart SH, Chan KM, Garibaldi LA. Pervasive human-driven decline of life on Earth points to the need for transformative change. Science. 2019 Dec 13;366(6471).

10. Daily GC. Nature's services (Vol. 3). Island Press, Washington, DC; 1997.

11. Costanza R, d'Arge R, De Groot R, Farber S, Grasso M, Hannon B, Limburg K, Naeem S, O'neill RV, Paruelo J, Raskin RG. The value of the world's ecosystem services and natural capital. nature. 1997 May;387(6630):253-60.

12. Fiedler AK, Landis DA, Wratten SD. Maximizing ecosystem services from conservation biological control: the role of habitat management. Biol Control. 2008 May 1;45(2):254-71.

13. Ings TC, Montoya JM, Bascompte J, Blüthgen N, Brown L, Dormann CF, Edwards F, Figueroa D, Jacob U, Jones JI, Lauridsen RB. Ecological networks beyond food webs. J Anim Ecol. 2009 Jan;78(1):253-69.

14. Kéfi S, Thébault E, Eklöf A, Lurgi M, Davis AJ, Kondoh M, Krumins JA. Toward multiplex ecological networks: Accounting for multiple interaction types to understand community structure and dynamics. In Adaptive Food Webs: Stability and Transitions of Real and Model Ecosystems 2017 Dec 21 (pp. 73-87). Cambridge University Press.

15. Fath BD, Scharler UM, Ulanowicz RE, Hannon B. Ecological network analysis: network construction. Ecol Model. 2007 Oct 24;208(1):49-55.

16. Fath B, Scharler U. Systems ecology: Ecological network analysis. In Encyclopedia of Ecology 2nd ed. 2018 (vol. 1 pp. 643-652). 
17. Friedler FE, Tarjan K, Huang YW, Fan LT. Combinatorial algorithms for process synthesis. Comput Chem Eng. 1992 May 1;16:S313-20.

18. Friedler F, Tarjan K, Huang YW, Fan LT. Graph-theoretic approach to process synthesis: Axioms and theorems. Chem Eng Sci. 1992 Jun 1;47(8):1973-88.

19. Friedler F, Aviso KB, Bertok B, Foo DC, Tan RR. Prospects and Challenges for Chemical Process Synthesis with P-graph. Curr Opin Chem Eng. 2019 Dec $1 ; 26: 58-64$.

20. P-Graph [Internet]. [cited 2020 Feb 10]. Available from: http://p-graph.com/

21. Friedler F, Tarjan K, Huang YW, Fan LT. Graph-theoretic approach to process synthesis: polynomial algorithm for maximal structure generation. Comput Chem Eng. 1993 Sep 1;17(9):929-42.

22. Friedler F, Varga JB, Feher E, Fan LT. Combinatorially accelerated branch-and-bound method for solving the MIP model of process network synthesis. In State of the art in global optimization 1996 (pp. 609-626). Springer, Boston, MA.

23. Tan RR, Aviso KB, Klemes JJ, Lam HL, Varbanov PS, Friedler F. Towards generalized process networks: Prospective new research frontiers for the P-graph framework. Chem Eng Trans. 2018 Aug 1;70:91-6.

24. Sterner RW, Elser J. Ecological stoichiometry: overview. In Encyclopedia of Ecology, Five-Volume Set 2008 Jan 1 (pp. 1101-1116). Elsevier Inc.. 


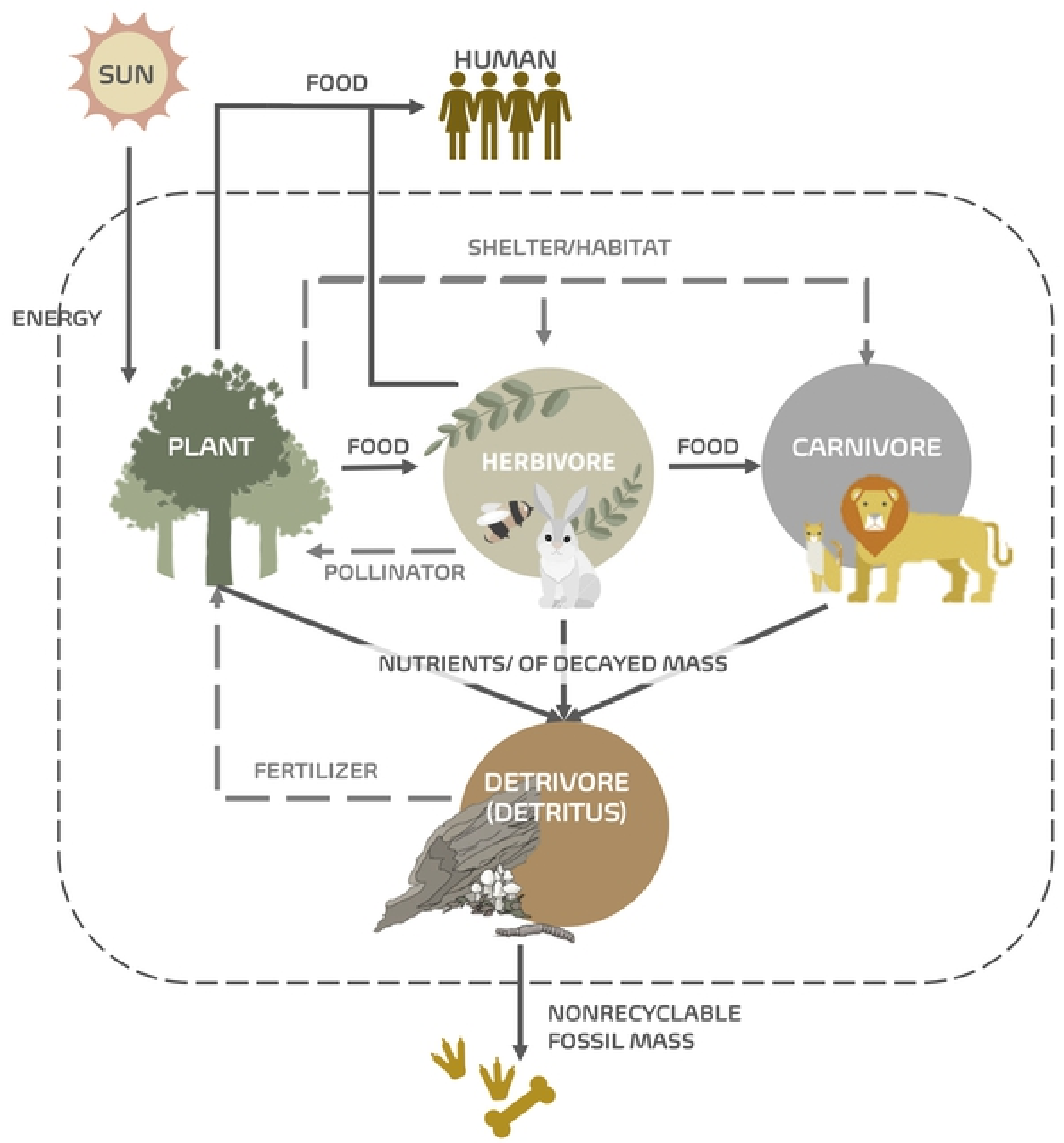

Figure 
(A)

Solar Energy

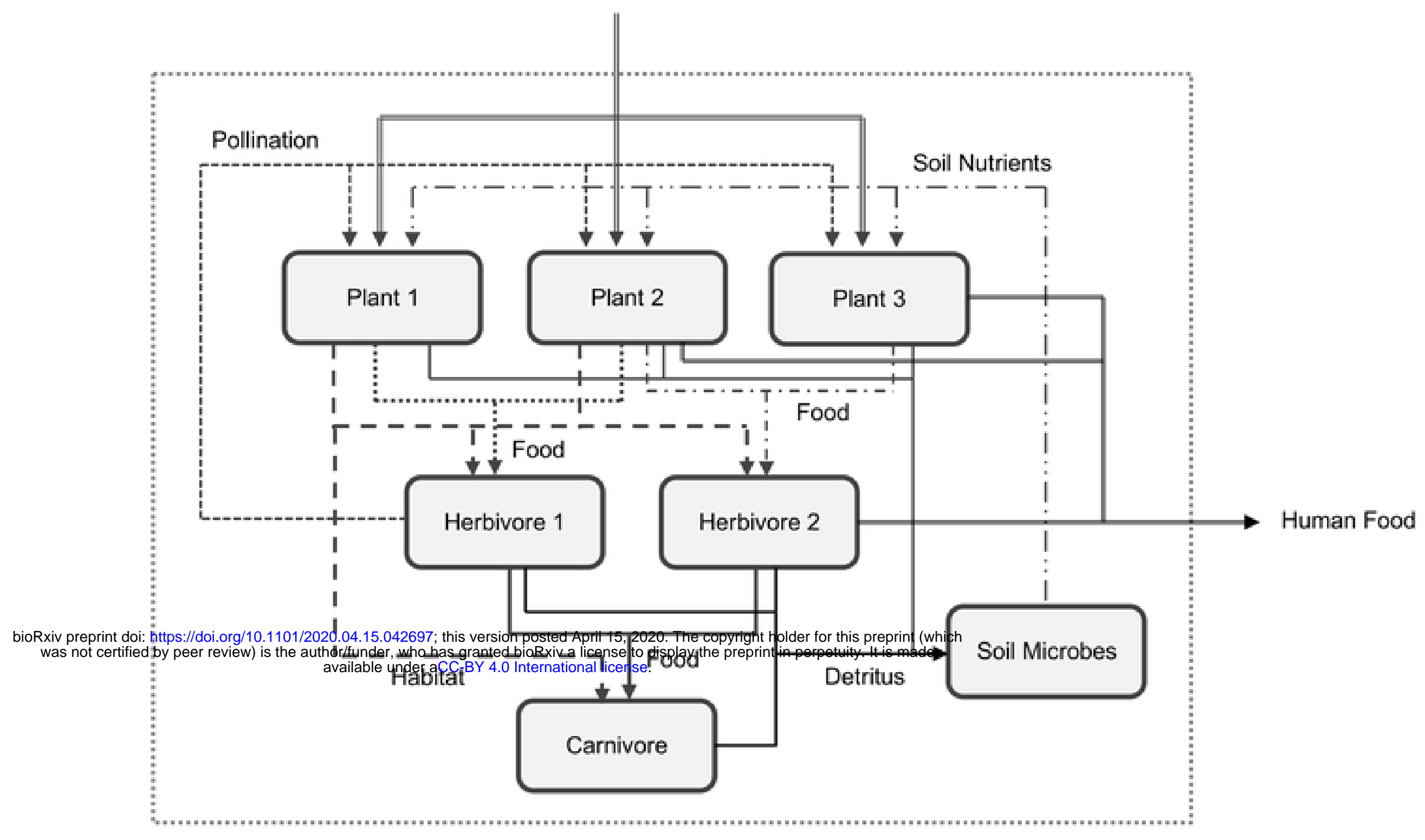

(B)

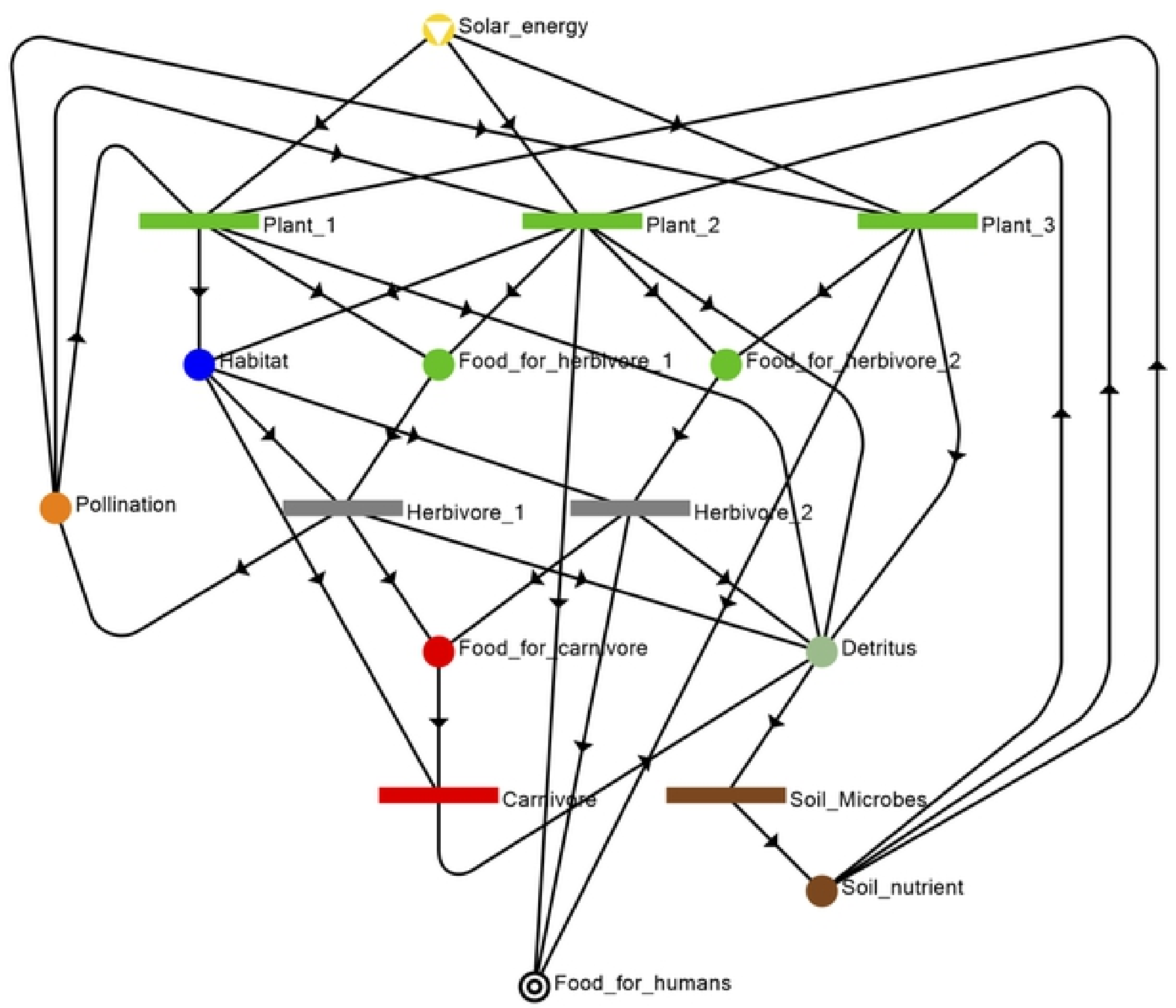

Figure 
(A)

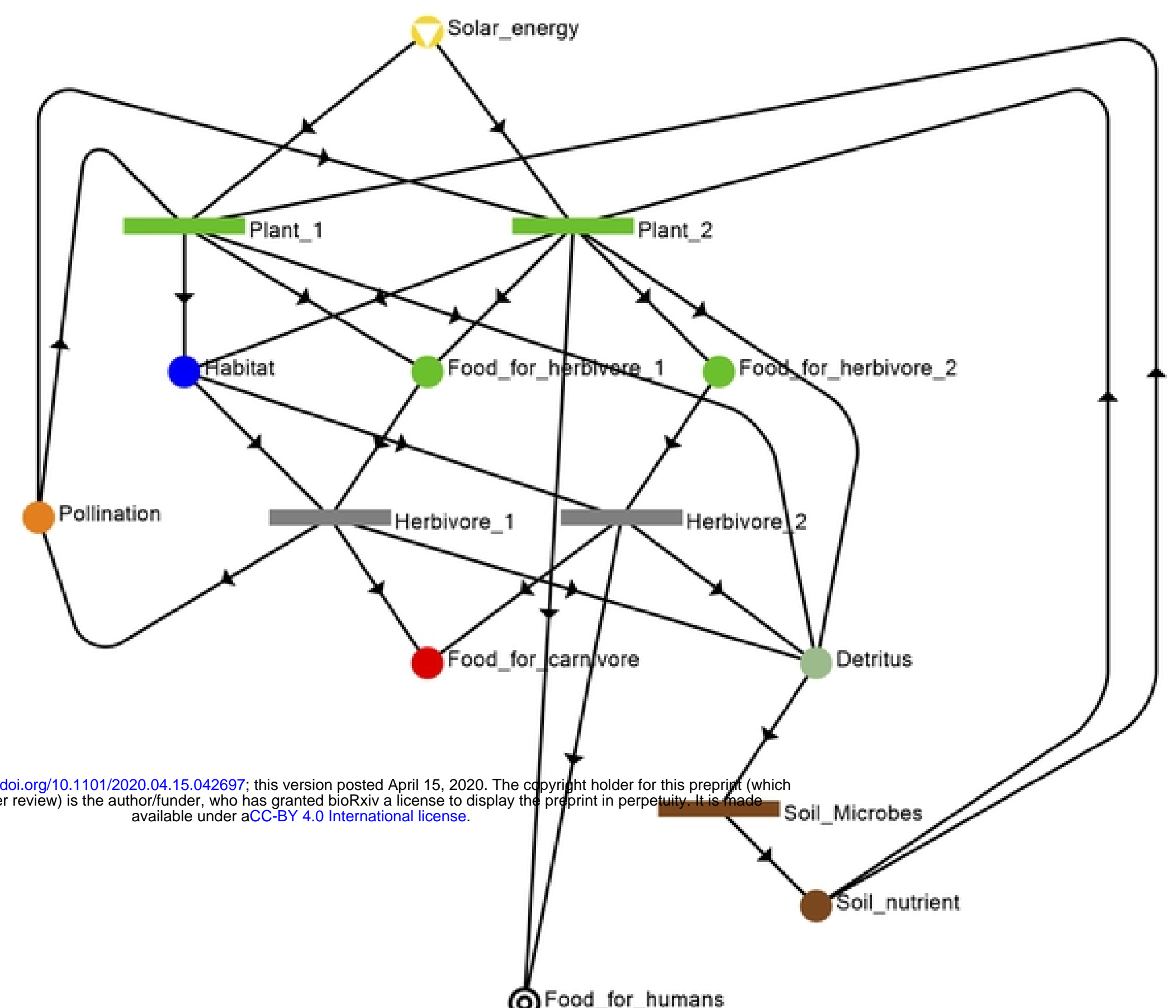

bioRxiv preprint doi: https://doi.org/10.1101/2020.04.15.042697; this version posted April 15, 2020. The cfpyright holder for this preprift (which

(.)Food_for_humans

(B)

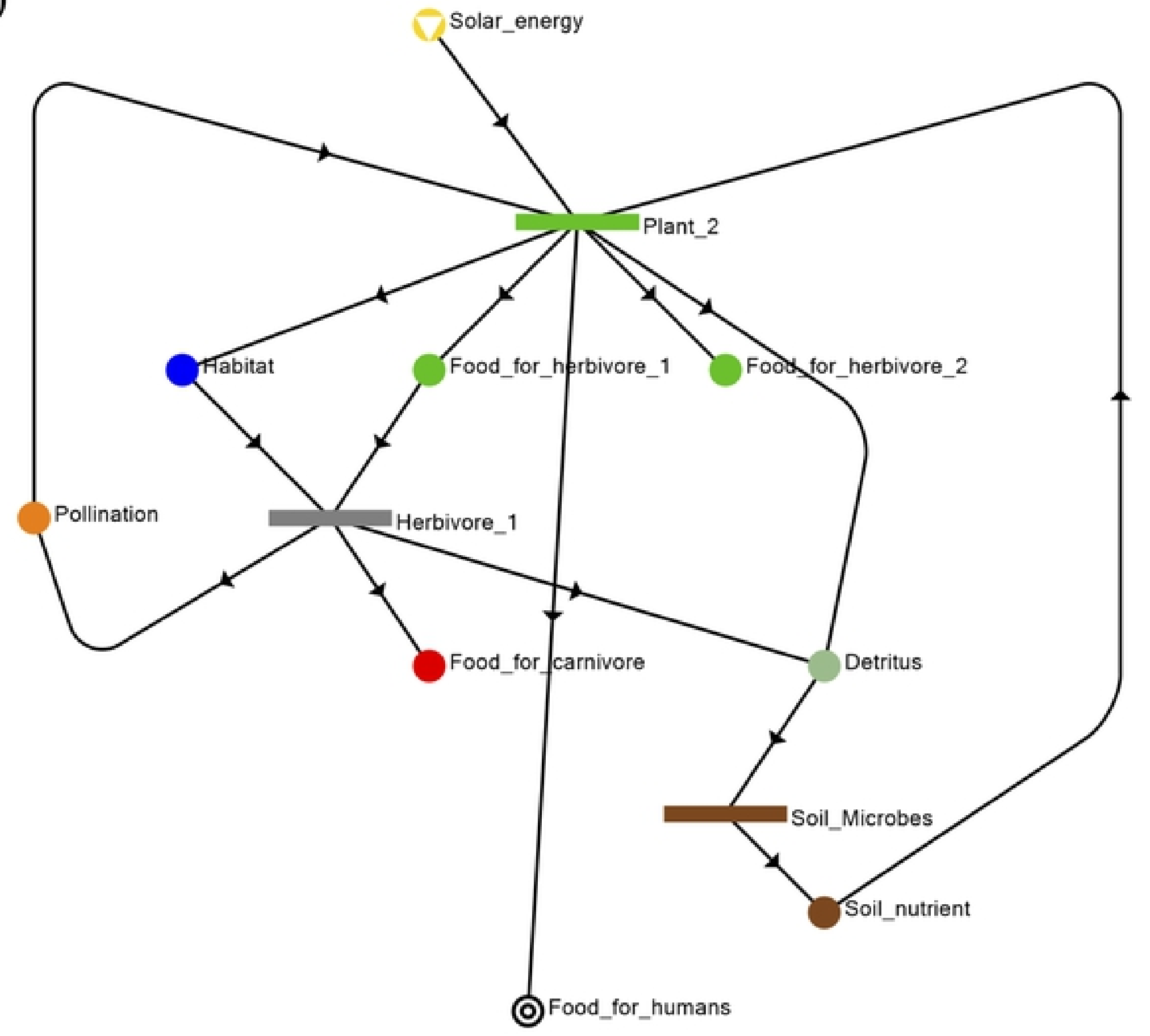

Figure 